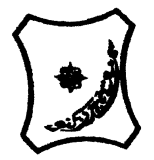

Bayero Journal of Pure and Applied Sciences, 12(1): 139 - 144 ISSN $2006-6996$

\title{
INCIDENCE OF EXTENDED SPECTRUM BETA-LACTAMASE PRODUCING Klebsiella pneumoniae AMONG PATIENTS WITH URINARY TRACT INFECTIONS IN KANO METROPOLIS NIGERIA
}

\author{
${ }^{1}$ Mohammed, A., ${ }^{2}$ Magashi, A.M. and ${ }^{2}$ Yushau, M. \\ ${ }^{1}$ Department of Pharmaceutical Microbiology and Biotechnology, Faculty of Pharmaceutical Sciences, \\ Bayero University Kano \\ ${ }^{2}$ Department of Microbiology, Faculty of Life Sciences, Bayero University Kano \\ Corresponding author: ayeeshapharm@yahoo.com +2348035897992
}

\begin{abstract}
Extended Spectrum Beta-Lactamase (ESBLS) production is one of the ways by which bacteria become resistant to antibiotics and pathogens of UTIs such as Klebsiella pneumoniae have been incriminated at global scale. This study was conducted to investigate the incidence of Extended Spectrum Beta lactamase producing Klebsiella pneumoniae from Urinary Tract Infections in Kano metropolis. The work involved One hundred and fourty seven $K$. Pneumoniae isolates obtained from patients with suspected urinary tract infections were studied from January to July 2017. The identity of the isolates was confirmed using Microgen ${ }^{T M}$ GnA + B-ID System. Antibiotic susceptibility testing was carried out using the Kirby-Bauer Disc Diffusion Technique. Screening for ESBLs production was done using Clinical Laboratory Standards Institute breakpoint. Suspected ESBLs producers were subjected to confirmation using Double Disc Synergy

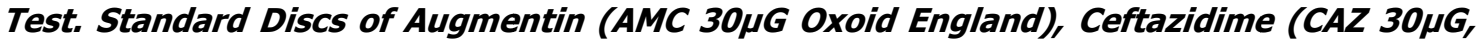

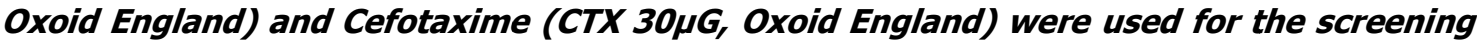
and confirmation. Accordingly, Multidrug Resistant $K$. pneumoniae were found to be 63.3\% and all were ESBLs producers. The Double Disc Synergy Test however confirmed $6.8 \%$ ESBLs producing $K$. pneumoniae. Antimicrobial sensitivity of the ESBLs producing organisms showed 100\% resistance to Augmentin, ceftriaxone, ceftazidime, cefotaxime while resistance to gentamicin was $91.5 \%$, chloramphenicol $23.4 \%$, Nitrofurantoin 61.7\%, Ciprofloxacin 93.6\% and cotrimoxazole 95.7\%. However, Imipenem was the most pharmacologically active drug. ESBL producing $K$. pneumoniae are incident in Kano and are resistant to commonly prescribed antibiotics. We, therefore, suggest screening and confirmation for ESBL in any attempt to treat UTIs due to such pathogens

Keywords

Extended Spectrum Beta Lactamases, $K$. pneumoniae, Urinary Tract Infection, Incidence, Kano
\end{abstract}

\section{INTRODUCTION}

Urinary Tract Infections are the most commonly reported hospital and community acquired infections, affecting more than half of the population at least once in their lifetime and has been associated with a high mortality rate. Majority of the causative agents in UTIs are Gram negative pathogens, primarily Enterobacteriaceae with Escherichia coli, Klebsiella pneumoniae and Proteus mirabilis being the main culprits (Tenney et al., 2018). $K$. pneumoniae has been named as one of the top three pathogens of international concern documented in the 2017 World Health
Organization's (WHO) Global Priority List of Antibiotic Resistant Bacteria to Guide Research, Discovery and Development of new antibiotics (WHO, 2017)

An increasing frequency in multi drug resistant K. pneumoniae that possess Extended Spectrum Beta Lactamases has been reported (Paterson and Bonomo, 2005). Multiple drug resistance has significantly increased among bacteria causing nosocomial infections and there is a growing concern for multi drug resistant Gramnegative bacteria which produce Extended Spectrum Beta Lactamases (ESBLs) (Coque et al., 2008). 
ESBLs are class A beta lactamases that hydrolyse penicillin, oxyimino-cephalosporins and monobactams. They are plasmid mediated enzymes with the most common types being TEM, SHV and CTX-M types. ESBLs are primarily produced by Enterobacteriaceae $E$. coli, Klebsiella pneumoniae and Klebsiella oxytoca (Paterson and Bonomo, 2005). An increase in antibiotic resistance among uropathogenic organisms to most common cephalosporins used in hospitals has been reported (Al janaby et al., 2017; Khalid et al., 2017)

The prevalence of ESBLs is increasingly being reported worldwide with it varying from one geographical location to another and being directly linked to the misuse and abuse of antibiotics (Jarlier et al., 1988). In many parts of the world, $10 \%-40 \%$ of strains of $\mathrm{E}$. coli and $\mathrm{K}$. pneumoniae express ESBLs (Rupp and Fey, 2003). In Nigeria, prevalence rates range from $5 \%$ to $44.3 \%$ as shown by (Olonitola et al., 2007; Olowe and Aboderin, 2010; Yushau et al., 2010; Akujobi and Ewuru, 2010; Ogefere et al., 2015; Mohammed et al., 2016; Giwa et al., 2018)

Several outbreaks of infection due to ESBL producing organisms have been described on every continent thereby posing as a challenge to infection control issues. Some initial outbreaks have been supplanted by endemicity leading to increase in patient morbidity and mortality (Paterson and Bonomo, 2005)

The aim of this study is to phenotypically investigate the incidence of Extended Spectrum Beta Lactamases (ESBLs) producing Klebsiella pneumoniae isolated from patients with UTIs in Kano Metropolis.

\section{MATERIALS AND METHODS}

A total of 1500 urine samples were collected from Aminu Kano Teaching Hospital, Murtala Muhammad Specialist Hospital and Muhammad Abdullahi Wase Specialist Hospital from In and Outpatients with suspected UTIs between January to July 2017 following approval from the Aminu Kano Teaching Hospital Ethical Committee and Kano State Ministry of Health.

Urine microscopy was carried out using a drop of uncentrifuged urine to determine significant pyuria. The samples were inoculated on Cysteine Lactose Electrolyte Deficient Agar and incubated at $37^{\circ} \mathrm{C}$ for $18-24$ hours. Discrete colonies were picked, and Gram staining was carried out. Further identification was done using Microgen ${ }^{T M} \mathrm{GnA}+\mathrm{B}$ ID biochemical identification system according to manufacturer's instructions. Klebsiella pneumoniae isolates that were obtained as a pure and predominant growth from the clinical specimen were only considered for the present study.

\section{Extended Spectrum Beta Lactamase Screening Test \\ Screening for ESBL production by disc diffusion test}

Resistance to cefotaxime ceftriaxone and ceftazidime was detected by disc diffusion test as recommended by (CLSI, 2017). From the pure cultures of bacteria grown overnight on MacConkey agar, a suspension matching 0.5 McFarland standard $\left(1.5 \times 10^{8} \mathrm{CFU} / \mathrm{ml}\right)$ was made in nutrient broth. Using sterile cotton swab, the bacteria was spread on Mueller Hinton agar to obtain a lawn culture. After allowing the plate to dry, the antibiotic discs mentioned above were placed on the surface and the plates incubated at $37^{\circ} \mathrm{C}$ for $18-24$ hours. Following growth, the diameter of the zone of inhibition around the disks were measured and recorded. The disc potency and zone diameters for inferring resistance were as follows; cefotaxime $(30 \mu \mathrm{g}) \leq 27 \mathrm{~mm}$, ceftriaxone $(30 \mu \mathrm{g}) \leq 25 \mathrm{~mm}$, ceftazidime $(30 \mu \mathrm{g})$. Resistance to at least one of the antibiotics will be considered as positive in the screening test for possible ESBL production (CLSI, 2017)

Confirmation of ESBL production by Double Disc Synergy Test (DDST)

Double Disc Synergy Test was carried out using 3 antibiotics, namely Amoxycillin-Clavulanic acid $(20 / 10 \mu \mathrm{g})$, Cefotaxime $(30 \mu \mathrm{g})$ and Ceftazidime $(30 \mu \mathrm{g})$. The discs were placed $25 \mathrm{~mm}$ (centre to centre of the discs) from Amoxycillin-Clavulanic acid on Mueller Hinton Agar. Enhancement of the zone of inhibition towards the clavulanate disc after 24 hours incubation at $37^{\circ} \mathrm{C}$ was considered indicative of a potential ESBL producer (Jarlier et al., 1988)

Antibiotic Sensitivity Testing of ESBL producing Klebsiella pneumoniae

This was carried out using Kirby-Bauer-CLSI modified Disc Agar Diffusion technique (DAD) (Hudzicki, 2009). One milliliter $(1.0 \mathrm{ml})$ of standardized overnight culture of each isolate (containing $10^{6} \mathrm{CFU} / \mathrm{ml}$ ) was used to flood the surface of Mueller Hinton Agar (MHA) plates and excess drained off and dried while the Petri dish lid was in place. The standard antibiotic discs (Gentamicin, Ceftazidime, Chloramphenical, Cefotaxime, Nitrofurantoin, Ciprofloxacin, Amoxicillin/Clavulanic acid, Imipenem, Ceftriaxone and Cotrimoxazole) were then aseptically placed at reasonable equidistance on the inoculated MHA plates and allowed to stand for $1 \mathrm{~h}$. 
The plates (prepared in duplicates for each isolate) were then incubated at $37^{\circ} \mathrm{C}$ for 18 hours. The diameter of the zones of inhibition produced by each antibiotic disc was measured and recorded.

\section{RESULTS AND DISCUSSION}

A total of $147 K$. pneumoniae isolates were obtained from the total samples examined. Table 1 shows the distribution of the organism across the different sampling sites and ESBL production with AKTH having the highest number of isolates followed by MMSH and MAWSH.This is similar to the study carried out by Kumurya and Sule (2016) at AKTH and Sule and Kumurya (2016) at MMSH where the number of $K$. pneumoniae isolates were $26.7 \%$ and $7 \%$ respectively. Several studies on uropathogens has shown Klebsiella pneumoniae to be the second most predominant pathogen after Escherichia coli (Ehinmidu 2003; El Mahmood 2009; Pondei et al., 2012; Iregbu and Nwajiobi-Princewill, 2013; Azekhueme et al., 2015; Mohammed et al., 2016; Onanuga et al., 2019).

In this study, ESBLs producing $K$. pneumoniae was $10.6 \%$. A higher prevalence was reported by Yushau et al (2010) at 25.5\%, in a study in India, nearly $40 \%$ of urinary isolates of $K$. pneumoniae were ESBL positive (Babypadmini and Appalaraju, 2004). ESBL producing $K$. pneumoniae were $54.4 \%$ in a study in Latin America (Aminazadeh et al., 2008). Mekki et al (2010) reported ESBL producing $K$. pneumoniae from the patients suffering from Urinary Tract Infections. Ejaz et al (2011) reported ESBL producing $K$. pneumoniae to be $71.7 \%$ in Pakistan. About $26.5 \%$ ESBLs producing $K$. pneumoniae has been reported in Ilorin (Fadeyi et al, 2016) and $40 \%$ and $40.7 \%$ ESBL producing $K$. pneumoniae has been reported in Zaria (Giwa et al., 2018) and Port Harcourt (Onanuga et al., 2019) respectively. These observed differences could be due to regional and attitudinal behaviour towards prescription and consumption of antibiotics especially the cephalosporins in both hospital and community settings.

Distribution of ESBLs across the hospitals showed that AKTH had the highest occurrence of ESBLs producing $K$. pneumoniae being $70 \%$. The spread of an ESBL variant can be facilitated by referral system where the presence of a single ESBL variant in a different centre may be imported by a patient on referral to another centre (Nordmann et al., 2009). This situation could hold for AKTH because it is a tertiary referral centre receiving patients from different parts of north western Nigeria.

\begin{tabular}{|c|c|c|c|c|c|}
\hline \multirow[t]{2}{*}{ Isolate } & \multicolumn{2}{|c|}{ Sampling site } & & ESBL Production & \multirow[b]{2}{*}{$\begin{array}{l}\text { Distribution of ESBL } \\
\text { production across the } \\
\text { sampling sites } \\
\text { AKTH MMSH MAWSH } \\
(\%)\end{array}$} \\
\hline & AKTH & $\begin{array}{l}\text { MMSH } \\
(\%)\end{array}$ & MAWSH & 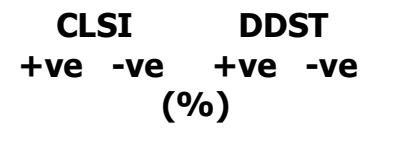 & \\
\hline $\begin{array}{l}K \\
\text { pneumoniae } \\
\mathrm{N}=147\end{array}$ & $\begin{array}{l}68 \\
(46.3)\end{array}$ & $\begin{array}{c}53 \\
(36.1)\end{array}$ & $\begin{array}{l}26 \\
(17.7)\end{array}$ & $\begin{array}{lccc}94 & 53 & 10 & 84 \\
(63.9) & (36.1) & (10.6) & (89.4)\end{array}$ & $\begin{array}{cc}2 & 1 \\
(20) & (10)\end{array}$ \\
\hline
\end{tabular}

Table 2 shows the Antibacterial Susceptibility pattern of ESBLs producing $K$. pneumoniae. A high level of resistance was observed among the isolates to Amoxicillin/Clavulanic acid and Cephalosporins has been widely reported in Nigeria and other parts of the world as expected because ESBLs production in Gram Negative bacteria is the key factor that confers resistance to beta lactam antibiotics except cephamycins and carbapenems (El Bouamri et al., 2015; Garbati et al., 2016; Pang et al., 2018; Onanuga et al., 2019). A high resistance of ESBLs producing $K$. pneumoniae to Gentamicin, Ciprofloxacin, Chloramphenical and Cotrimoxazole (70-100\%) has also been reported by Onanuga et al. (2019) which poses a significant problem to the treatment of urinary tract infections with this commonly used antibiotics thereby narrowing the choice of antimicrobial agents effective against ESBLs producing organisms (Paterson et al., 2001). Multi drug resistance among ESBLs producing $K$. pneumoniae in this study is similar to the findings of Eshetie et al., 2015 in Ethopia who reported $87.4 \%$ MDR $K$. pneumoniae and Onanuga et al. (2019) also reported $100 \%$ while Giwa et al. (2018) reported a lesser value of $40 \%$.

Imipenem was the most effective antibiotic with $100 \%$ sensitivity. A similar result has been reported by Ejikeugwu et al. (2012) and Igbinoba and Osazuwa (2012) in Nigeria. 
However, carbapenem resistance has been reported widely as an increasing public health problem (Garbati et al., 2016; Pang et al., 2018). Effectiveness of Imipenem could be due to its late arrival in the Nigerian market therefore to ensure its continued relevance a coordinated rationale usage must be implemented.

Table 2: Antibacterial Susceptibility pattern of ESBLs producing $K$. pneumoniae $(\mathrm{N}=10)$

\begin{tabular}{|c|c|c|}
\hline Antibiotics & Sensitive (\%) & Resistant (\%) \\
\hline Gentamicin $(10 \mu \mathrm{g})$ & $1(10)$ & $9(90)$ \\
\hline Ceftazidime $(30 \mu \mathrm{g})$ & $0(0)$ & $10(100)$ \\
\hline Chloramphenical $(30 \mu \mathrm{g})$ & $3(30)$ & $7(70)$ \\
\hline Cefotaxime $(30 \mu \mathrm{g})$ & $0(0)$ & $10(100)$ \\
\hline Nitrofurantoin $(300 \mu \mathrm{g})$ & $6(60)$ & $4(40)$ \\
\hline Ciprofloxacin $(5 \mu \mathrm{g})$ & $2(20)$ & $8(80)$ \\
\hline 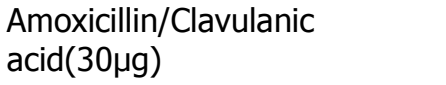 & $0(0)$ & $10(100)$ \\
\hline Imipenem $(10 \mu \mathrm{g})$ & $10(100)$ & $0(0)$ \\
\hline 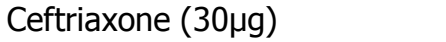 & $0(0)$ & $10(100)$ \\
\hline Cotrimoxazole $(1.25 / 23.75 \mu \mathrm{g})$ & $0(0)$ & $10(100)$ \\
\hline
\end{tabular}

\section{CONCLUSION}

The incidence of phenotypically expressed ESBLs producing $K$. pneumoniae is high and they are generally multi drug resistant. Carbapenems remain the most useful therapy for infections caused by this organism. A functional antibiotic prescription policy that involves the rationale use

\section{REFERENCES}

Akujobi, C. N., and Ewuru, C. P. (2010). Detection of extended spectrum betalactamases in gram negative bacilli from clinical specimens in a teaching hospital in South eastern Nigeria. Nigerian Medical Journal, 51(4), 141.

Aljanaby, A. A. J., and Alhasani, A. H. A. (2016). Virulence factors and antibiotic susceptibility patterns of multidrug resistance Klebsiella pneumoniae isolated from different clinical infections. African Journal of Microbiology Research, 10(22), 829-843.

Aminzadeh, Z., Sadat, K.M and Shabani, M (2008). Bacteriuria by extended spectrum Beta-lactamase-producing $E$. coli and Klebsiella pneumoniae: isolates in a governmental hospital in South of Tehran, Iran. Iran Journal of Kidney Diseases. 2(4), 197-200

Azekhueme, I., Moses, A. E., \& Abbey, S. D. (2015). Extended spectrum betalactamases in clinical isolates of Escherichia coli and Klebsiella pneumoniae from University of Uyo Teaching Hospital, Uyo-Nigeria. Journal of Advances in Medical and Pharmaceutical Sciences, 2(3), 117-125.

Babypadmini, S. and Appalaraju, B. (2004). Extended spectrum-lactamases in of carbapenems needs to be implemented to prevent failure. It is recommended that a continued surveillance using well-equipped laboratories for prompt detection and reporting of ESBLs producing $K$. pneumoniae should be implemented as well as making it a routine procedure in hospitals

urinary isolates of Escherichia coli and Klebsiella pneumoniae-prevalence and susceptibility pattern in a tertiary care hospital. Indian Journal of Medical Microbiology, 22(3), 172.

CLSI (2017). Clinical and Laboratory Standards Institute. Performance Standards for Antimicrobial Susceptibility Testing: Twenty-first Informational Supplement M100-S20. Wayne, PA, USA

Coque, T. M., Baquero, F., \& Canton, R. (2008). Increasing prevalence of ESBLproducing Enterobacteriaceae in Europe. Eurosurveillance, 13(47), 19044.

Ehinmidu, J.O. (2003). Antibiotics susceptibility patterns of urine bacterial isolates in Zaria, Nigeria. Journal of Tropical Pharmaceutical Research 2(1), 223-228.

Ejaz, H., Zafa, A., Mahmood, S. and Javed, M. M. (2011). Urinary tract infections caused by extended spectrum $\beta$ lactamase (ESBL) producing Escherichia coli and Klebsiella pneumoniae. African Journal of Biotechnology, 10(73), 16661-16666.

Ejikeugwu P.C., Ugwu C.M., Araka C.O., Gugu T.H., Iroha I.R and Adikwu M.U(2012). Imipenem and meropenem resistance amongst ESBLs producing 142 Escherichia coli and Klebsiella 
pneumoniae clinical isolates. International Research Journal of Microbiology 3(10): 339-344

El Bouamri, M. C., Arsalane, L., El Kamouni, Y. and Zouhair, S. (2015). Antimicrobial susceptibility of urinary Klebsiella pneumoniae and the emergence of carbapenem-resistant strains: A retrospective study from a university hospital in Morocco, North Africa. African Journal of Urology, 21(1), 36-40.

El-Mahmood, A.M., Atimi, A.T., Tirmidhi, B. and Mohammed, A.(2009) Antimicrobial susceptibility of some quinolone antibiotics against some urinary tract pathogens in a tertiary hospital, Yola, Adamawa State, Nigeria. Journal of Clinical Medicine and Research 1(1), 2634.

Eshetie, S., Unakal, C., Gelaw, A., Ayelign, B., Endris, M., and Moges, F. (2015). Multidrug resistant and carbapenemase producing Enterobacteriaceae among patients with urinary tract infection at referral Hospital, Northwest Ethiopia. Antimicrobial resistance and infection control, 4(1), 12.

Fadeyi, A., Zumuk, C. P., Raheem, R. A., Nwabuisi, C. and Desalu, O. O. (2016). Prevalence and antibiotic susceptibility pattern of ESBL producing Klebsiellae isolated from clinical specimens in a Nigerian tertiary hospital. African Journal of Infectious Diseases, 10(1), 32-37.

Garbati, M. A., Sakkijha, H., \& Abushaheen, A. (2016). Infections due to carbapenem resistant Enterobacteriaceae among Saudi Arabian hospitalized patients: a matched case-control study. BioMed research international, 2016.

Giwa, F.J., Ige, O.T., Haruna, D.M., Yaqub, Y., Lamido, T.Z. and Usman, S.Y. (2018) Extended-Spectrum beta-lactamase production and antimicrobial susceptibility pattern of uropathogens in a Tertiary Hospital in Northwestern Nigeria. Annals of Tropical Pathology 9:11-6.

Hudzicki Jan. (2009) Kirby-bauer disk diffusion susceptibility test protocol

Igbinoba, A. O. and Osazuwa, F. (2012). Zero resistance to the carbapenems among extended spectrum betalactamase producing Klebsiella pneumoniae in a Nigerian university hospital. Int. J. Biol., Pharmacy and Allied Sciences, 1(1), 7983.
Iregbu, K. C. and Nwajiobi-Princewill, P. I. (2013). Urinary tract infections in a tertiary hospital in Abuja, Nigeria. African Journal of Clinical and Experimental Microbiology, 14(3), 169173.

Jarlier, V., Nicolas, M. H., Fournier, G. and Philippon, A. (1988). Extended broadspectrum $\beta$-lactamases conferring transferable resistance to newer $\beta$ lactam agents in Enterobacteriaceae: hospital prevalence and susceptibility patterns. Clinical Infectious Diseases, 10(4), 867-878.

Khalid, H.M., Yousif, S.Y. and Jubrael, J.M.S. (2017). Bacteriological and Molecular characterization of extended spectrum betalactamases in clinical isolates of Klebsiella pneumoniae isolated from Kurdistan region, Iraq. Science Journal of University of Zakho, 1(1), 158-163

Kumurya, A.S and Sule, H (2016). The prevalence of Escherichia coli Causing Urinary Tract Infections in Aminu Kano Teaching Hospital, Kano, Nigeria. World Journal of Microbiology, 2(2): 033-037

Mekki, A. H., Hassan, A. N. and Elsayed, D. E. M. (2010). Extended spectrum beta lactamases among multi drug resistant Escherichia coli and Klebsiella species causing urinary tract infections in Khartoum. African Journal of Bacteriology Research, 2(3), 18-21

Mohammed, Y., Gadzama, G. B., Zailani, S. B., and Aboderin, A. O. (2016). Characterization of extended-spectrum beta-lactamase from Escherichia coli and Klebsiella species from North Eastern Nigeria. Journal of clinical and diagnostic research: JCDR, 10(2), DC07.

Nordmann, P., Cuzon, G and Naas, T. (2009). The real threat of Klebsiella pneumoniae carbapenemase-producing bacteria. Lancet Infectious Diseases, 9(4), 228236.

Ogefere, H. O., Aigbiremwen, P. A., and Omoregie, R. (2015). Extendedspectrum beta-lactamase (ESBL)producing Gram-negative isolates from urine and wound specimens in a tertiary health facility in southern Nigeria. Tropical Journal of Pharmaceutical Research, 14(6), 10891094. 
Olonitola, O. S., Olayinka, A. T., Inabo, H. I., and Shaibo, A. M. (2007). Production of extended spectrum beta-lactamases of urinary isolates of Escherichia coli and Klesiella pneumoniae in Ahmadu Bello University Teaching Hospital, Zaria, Nigeria. International Journal of Biological and Chemical Sciences, 1(2), 181-185.

Olowe, O. A., and Aboderin, B. W. (2010). Detection of extended spectrum $\beta$ lactamase producing strains of (Escherichia coli) and (Klebsiella sp.) in a tertiary health centre in Ogun State. International Journal of Tropical Medicine, 5(3), 62-64.

Onanuga, A., Vincent, C. H., \& Eboh, D. D. Carbapenem Resistance among Extended Spectrum Beta-Lactamases Producing Escherichia coli and Klebsiella pneumoniae isolates from Patents with Urinary Tract Infections in PortHarcourt, Nigeria. Nigerian Journal of Pharmaceutical and Applied Science Research, 8(1), 16-23.

Pang, F., Jia, X. Q., Zhao, Q. G., \& Zhang, Y. (2018). Factors associated to prevalence and treatment of carbapenem-resistant Enterobacteriaceae infections: a seven years retrospective study in three tertiary care hospitals. Annals of clinical microbiology and antimicrobials, 1入1), 13.

Paterson, D. L and Bonomo, R. A. (2005). Extended-spectrum beta-lactamases: a clinical update. Clinical Microbiology Reviews, 18(4), 657-686.

Paterson, D. L., Ko, W. C., Von Gottberg, A., Casellas, J. M., Mulazimoglu, L., Klugman, K. P., ..and Victor, L. Y. (2001). Outcome of cephalosporin treatment for serious infections due to apparently susceptible organisms producing extended-spectrum $\beta$ lactamases: implications for the clinical microbiology laboratory. Journal of clinical microbiology, 39(6), 2206-2212.

Pondei, K., Oladapo, O. and Kunle-Olowu, O. E. (2012). Anti-microbial susceptibility pattern of micro-organisms associated with urinary tract infections in a tertiary health institution in the Niger Delta Region of Nigeria. African Journal of Microbiology Research, 6(23), 49764982.

Rupp, M. E., and Fey, P. D. (2003). Extended spectrum $\beta$-lactamase (ESBL)-producing Enterobacteriaceae. Drugs, 63(4), 353365.

Sule, H. and Kumurya, A. S. (2016). The Prevalence of Klebsiella Species Causing Urinary Tract Infections in Murtala Muhammad Specialist Hospital, Kano, Nigeria. American Journal of Biomedical and Life Sciences, 4(2), 11-15.

Tenney, J., Hudson, N., Alnifaidy, H., Li, J. T. C. and Fung, K. H. (2018). Risk factors for acquiring multidrug-resistant organisms in urinary tract infections: a systematic literature review. Saudi pharmaceutical journal, 26(5), 678-684.

WHO Global Priority List of Antibiotic-Resistant Bacteria to Guide Research, Discovery, and Development of New Antibiotics. [(accessed on 27 February 2017)]; Available

online: https://www.who.int/medicines/ publications/global-priority-list-antibioticresistant-bacteria/en/

Yusha'u, M., Aliyu, H. M., Kumurya, A.S. and Suleiman, K. (2010) Prevalence of extended spectrum $\beta$-lactamases (ESBLs) among Enterobacteriaceae in Murtala Mohammed Specialist Hospital, Kano, Nigeria. Bayero Journal of Pure and Applied sciences, 3(1): 169- 172. 